\title{
Health care practices influencing health promotion in urban black women in Tshwane
}

\author{
SCD Wright, D Tech Nursing \\ Senior lecturer, Adelaide Tambo School of Nursing Science Tshwane University of Technology \\ JE Maree, D Cur (Pret) \\ Head of Department, Adelaide Tambo School of Nursing Science Tshwane University of Technology
}

Keywords:

Health care practices, health promotion, black women.

\section{Correspondence address:}

Dr. Susanna CD Wright

Adelaide Tambo School of Nursing

Science

Tshwane University of Technology

Private bag X680,

Pretoria, 0001

Tel : (012) 382-50470

Fax : (012) 382-5033

E-mail: wrightscd@tut.ac.za

\section{Abstract: Curationis 31(3): 36-43}

Health promotion is a multifaceted activity. Women and children are particularly vulnerable regarding access to quality health care, with young African women reportedly the poorest and most economically marginalised and least educated sector in South Africa. Understanding the context within which a person lives is an essential component in the health educator's teaching strategy. Understanding urban black women's health care practices will enable health promoters to develop interventions that are successful. The problem investigated was to gain an understanding of the health care practices of urban black women that could influence health promotion activities. The design was qualitative exploratory. The respondents were women living in an urban township in Tshwane, South Africa. The sampling method was convenient and purposive and the sample size was determined by saturation of the data. Data was gathered through semi-structured interviews using six specific themes and the analysed using open coding. The results indicated that the social environment created by the registered nurses in the primary health influenced the health care practices of the women negatively. Practices regarding the seriousness of a health problem suggest a possible reason for late admission of a person with a serious health problem.

\section{Introduction}

Health promotion is a multifaceted activity. Women and children are particularly vulnerable regarding access to quality health care, with young African women reportedly the poorest and most economically marginalised and least educated sector in South Africa (Gilbert \& Walker, 2002:152). A healthy life for a mother and a child is essentially dependent on the level of knowledge and education of the mother as the recipient of health care messages. This in itself would be the key factor for successful healthy living (Bunton, Nettleton \& Burrows, 1996:2).

The multifaceted nature of health promotion can be illustrated by the example of educating a mother on the prevention of dehydration to treat a child with gastro-enteritis, without knowledge of the prevailing feeding practises, the socio-economic circumstances or the environment in which the family lives. Not knowing or improving these circumstances will lead to little or no change in the health status of the child. If dehydration prevention is the only message, the gastro-enteritis will reoccur. Only if all of these complex factors in the household are taken into account and improved, can the lives both mother and child be conducive to healthy living. In short, health promotion should embrace all facets of the living reality of the mother and child.

It is accepted in literature that a child's 
health is dependent upon the mother's educational level (Gilbert \& Walker, 2002:151). From a health perspective, being marginalised and uneducated, places the mother and the child in a very vulnerable position. On a personal level, for the mother to be knowledgeable, she must be taught. Inherent in teaching is readiness and willingness to learn, taking into consideration factors such as language, culture, literacy levels, previous knowledge, trust, time, space and interpersonal relationships. Understanding the context within which the mother lives is an essential component in the educator's teaching strategy. Also, being taught in ways that are insensitive to a person's expectations and values will not result in changes in her feeding or health care practices.

According to Kelly and Charlton (1996:90), to understand human behaviour, there should be sufficient understanding of social structure, the individual's comprehension of this structure and how it impinges on their lives. The person must be set into context. Sharp (1988:15) in an essay on 'Constructing social reality', writes that the fact that people identify with a specific group, for example Afrikaner, Zulu or Tswana, does not entitle one to suppose that the groups in question are all-encompassing, and constitute the one and only identity that their members will acknowledge. The classic error is to presume that people, who identify themselves as 'Zulu' for example, will be the same and that the label of 'Zulu' says all that is to be said about them. Every person is a unique individual in a social context.

A range of factors delineates the social context. Culture is such a factor. According to Gilbert and Walker (2002:46), all communities understand health in terms of their culture. Associated with the values and beliefs about health, every community has set ways of maintaining health, preventing illness and treating the sick. The problem is that the use of the term 'community' according to the author indicates a homogeneous unit, but modern communities are geographically defined and inherently heterogeneous. The geographical location of a community also has an explicit influence on a community's health. Educational efforts and preventive measures therefore need to take cognisance of the social and cultural factors in communities.

Understanding urban black women's health care practices and their rationale for acting in a specific way will enable health promoters to develop interventions that are successful. Of specific interest are their ways of doing within their culture, namely on when a health problem occurs, on how decisions are made, on when a health problem is considered as serious and on how long symptoms should be present before action is taken. Another factor of particular consequence for health promotion is the phenomenon of personal acquiescence when a person is in the presence of an authority figure. The practice of saying 'yes' when health education is given without the person understanding at all, negates any positive effect the health education could have had. Health promoters need to have an understanding of the possible differences and influence of health care practices in a community. Some stereotypes exist from the past. If the women in a specific culture were known to behave in a certain way when there was a sick person in the household, the behaviour is regarded as fixed. Influences such as urbanisation and westernisation, and living in a global context and a heterogeneous society must influence individual's ways of doing.

Communities are not culturally homogeneous and thus there may ve various ways of doing within a community. An outline of the various practices concerning health in a community will enable health promoters to develop strategies based on this knowledge and understanding. One definition of health promotion that is often used is enabling people to increase control over, and to improve, their health (Dennill, King \& Swanepoel, 2004:13). The 'people' are adults, not children. A child does not have the knowledge or capacity to make decisions about his own health. The role of the mother in health related matters in the household are therefore important. Due to the tradition that the women in the extended family are the decision makers concerning health problems, their ways of doing will influence the decisions made when a health problem occur in the household.

\section{Research problem}

The research problem investigated in this study was the lack of understanding of the health care practices of urban black women that could influence health promotion activities.

The purpose of the study was to determine the health care practices of urban black women that could influence health promotion activities. Without understanding local health care practices, registered professional nurses will not be able to develop educational strategies and health promotion interventions congruent with or the local health care practices. Health promotion will be ineffective if local health care practices are not taken into consideration.

\section{Research Design and Method}

The strategy for the study was exploratory (Burns \& Grove, 2005:356). The context of the study was GaRankuwa, a township $20 \mathrm{~km}$ north of Tshwane in South Africa. The population is heterogeneous in terms of culture, socio-economic characteristics and health related needs.

\section{Design}

The research design was qualitative exploratory. According to Burns and Grove (2005:27), qualitative research is any kind of research that produces findings not arrived at by means of statistical procedures or other means of quantification. It can refer to research about person's lives, stories, behaviour, but also about organisational functioning, social movements, or interactional relationships. The health care practices of the women of an urban community were explored. The qualitative paradigm is applicable, as there was no intent to generalise the findings to a larger context.

\section{Population}

The respondents for the study were women living in an urban township area. The women of the households were included in the study due to their decision making role in health related matters. The criteria for inclusion in the study were:

- willingness to participate;
married woman with children;
and
living in the chosen area.

Being married gave the women an accepted social position within the 


\begin{tabular}{|c|c|}
\hline Principle & Application in study \\
\hline Prolonged engagement & $\begin{array}{l}\text { The field worker interviewing the respondent at her home in her own language will } \\
\text { ensure a trusting relationship. }\end{array}$ \\
\hline $\begin{array}{l}\text { Referential adequacy } \\
\text { Source data }\end{array}$ & $\begin{array}{l}\text { Enough data was gathered to ensure saturation for each question individually. } \\
\text { Referential adequacy was ensured. }\end{array}$ \\
\hline Consistency & An interview schedule was used as the basis for the interviews. \\
\hline Field Worker & $\begin{array}{l}\text { The field worker had a health care background. She was trained to do qualitative } \\
\text { interviews and a quality check was done throughout the data gathering. }\end{array}$ \\
\hline Audit trail & $\begin{array}{l}\text { An audit trail was developed to serve as proof that the results are grounded in the } \\
\text { data. Initial and final categories were traceable to the interview that generated the } \\
\text { category. A record of the key decisions made during the data analysis was kept. }\end{array}$ \\
\hline Dependability audit & $\begin{array}{l}\text { The raw data of the interview was coded, audited, and archived to permit checking } \\
\text { of the findings against the raw data.Tesch's approach was used to analyse the } \\
\text { data. }\end{array}$ \\
\hline Peer examination & $\begin{array}{l}\text { The research proposal was evaluated and accepted by the Faculty Research } \\
\text { Committee and the Ethics Committee of the Tshwane University of Technology } \\
\text { before implementation of the study. }\end{array}$ \\
\hline
\end{tabular}

extended family and she would be able to use the social resources in the family, whether it was health related knowledge or needing a decision for a health related action. The sampling method was convenient and purposive and the sample size was determined by saturation of the data (Lincoln \& Guba, 1985, chapter 11). The sample size was 50 respondents. Data analysis was done concurrently with the data gathering to be able to determine when the data was saturated.

\section{Data gathering}

Due to the qualitative nature of the data needed for the study, semi-structured interviews (Burns \& Grove, 2005:396) were conducted to gather the data. To guide the interviews, an interview schedule with six themes was developed. The themes were as follows:

1. Personal acquiescence with a person in an authoritative position e.g. a health care worker.

2. Behavioural patterns when a person in the household needs health care.

3. Assessment of the seriousness of a health problem.

4. Help seeking preferences for health related problems.

5. Decision making processes when a person needs health care.

6. Concept of time in health related problems.

The interviews were conducted at the participant's home during the day. As this was the natural setting of the participant, she was comfortable and at ease. One trained field worker, a registered nurse, was employed to gather the data. The field worker was more suitable due to the language needs of the participants and to improve rapport between the participant and the research team. As the field worker was the primary data instrument in the study, a trusting relationship was important and thus realised.

The research protocol was presented to and approved by the Ethics Committee of the Institution. Informed consent was obtained before the interview. The field worker completed a prepared form with the themes during the interview. The field notes were numbered sequentially to ensure anonymity and confidentiality.

\section{Data analysis}

Open coding using Tesch's approach (Creswell, 1994:135) was done. Initial and final categories were generated from the data. The data was analysed in terms of health care practices that could influence health promotion. Data analysis commenced after the first interview. Saturation was obtained for each question individually.

\section{Trustworthiness}

Trustworthiness was established according to the principles promoted by Lincoln and Guba (1985) and presented in Table 1.

Appropriate ethical principles were upheld in the study. Informed consent was obtained from the participants before commencement of the semi-structured interviews. Anonymity and confidentiality was ensured through numbering the transcribed interviews sequentially. Participation was voluntary and the participants could withdraw from the study at any point during the interview.

\section{Results of the study}

The results will be reported in two sections, namely a demographic overview of the group interviewed and the health care practice in terms of the six interview themes.

\section{Demographic overview of the respondents}

The group consisted of 50 married 
women with children, living in an urban area in Tshwane, South Africa. The ages ranged from early twenties to eighties with $50 \%$ of the group below 50 years of age. Concerning literacy, $54 \%$ had at least a secondary school or tertiary education. The unemployment figure was high in the group with seven out of ten unemployed. Twenty percent of the group lived in informal housing which indicates a shack with no electricity, sanitation or running water. The average number of occupants per household was five living in a four-roomed house. The group consisted mainly of Setswana speaking people $(82 \%)$.

\section{Health care practices}

The health care practices will be described in terms of the six interview themes.

Theme 1: Personal acquiescence with a person in an authoritative position, for example, a health care worker

Data saturation was obtained after 29 interviews. Two main categories were developed from the data: willingness to obtain health information and unwillingness to obtain health information.

\section{- Willingness to obtain health \\ information}

The majority of the respondents $(72 \%)$ reported that they would ask questions if they did not understand. The respondents contextualised the question in their local clinic without being prompted to do so. Asking questions about health issues were however, not always without risk. The registered nurses working in the clinic had a definite influence on the respondents' willingness to ask questions. Some of the respondents who were willing to ask questions did so regardless of the registered nurses attitude towards them.

"I ask when I don't understand though the sisters in the clinics are very short tempered."

"I don't care if they (the registered nurses) become rude to me but as long as they give me the information I need"

Some respondents indicated that they have learned to avoid the registered nurses who are rude when they need information.

"I ask questions if I do not understand
Figure 1 : Behavioural patterns when a person in the household needs health care

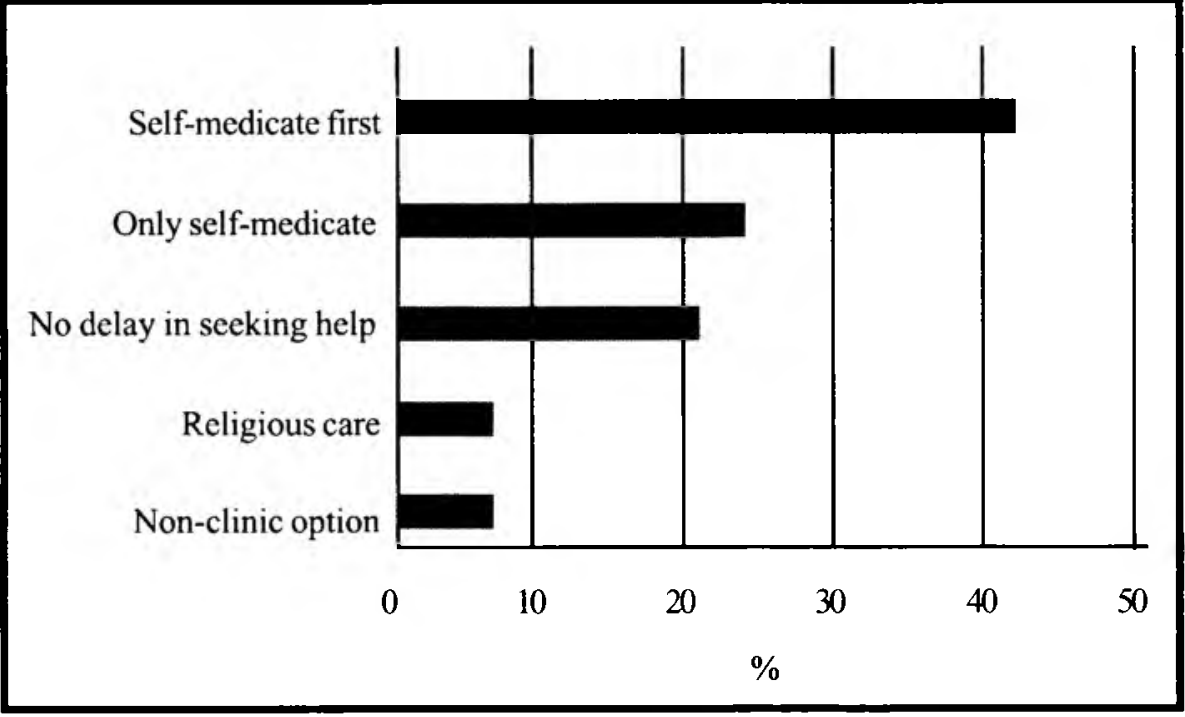

though I know that some of the nurses are easily irritable but I ask the ones I feel comfortable with."

"I know that nurses are rude and cruel but I will ignore that and ask the question. I'm not scared."

Another factor that became clear from the interviews was that, though the registered nurses did give health education, their focus was on HIV and the other conditions and diseases were neglected. According to a respondent "Even though the sisters give a lot of health education about Aids, nothing else ... without answers of the other diseases like arthritis."

\section{- Unwillingness to obtain health} information

About a third of the women were not willing to ask questions even if they needed additional information. Two factors are indicated as the reason for their unwillingness to ask questions. These factors are the attitude of the registered nurses in the clinic and the group setting in which health education takes place.

Several respondents indicated that they actually fear the registered nurses and would not ask a question at all. One respondent said, "I do not ask questions because some of the nurses are very rude and they scream at me." Other respondents were very clear that they will not ask any questions, for instance: "The way I am being treated by the nurses leads me to end up being scared of the nurses."

One respondent mentioned that she does not ask questions when she is part of a group because she is too shy but that she will ask if she is in a one on one situation.

Theme 2: Behavioural patterns when a person in the household needs health care

Data saturation was obtained after 45 interviews.

The frequency of the five main categories is presented in Figure 1.

\section{- Self-medicate first before} seeking help in the health care system Almost half of the group (43\%) would self-medicate first before they would seek help in the health care system, traditional or biomedical. One respondent reported that they would not just go to the clinic; they would try every medication possible like Panado $($ (paracetamol). They would use cooking oil mixed with vinegar and give that to a child for coughing. Lingana (herb) is also given for flu. The woman would treat herself by inducing vomiting to reduce headache and she would use lukewarm water to treat the headache. Another respondent reported that they would use herbs as they are told to do so by the nurses because they should not bother the nurses with "little complaints". A third respondent said the whole family used Lingana, Wenreid and cooked Senokolo (herbs) as these would open up the chest. A mother reported that she would use gripe water and Panado $B$ for fever as there is no need for a child to go to the clinic in case of a fever. If her husband is sick, he would go straight to the doctor but she looks after the 
children's health.

- $\quad$ Only self-medicate $\mathbf{( 2 5 \% )}$

One in four women reported that they would preferably only self-medicate. One report is as follows: "... in case of

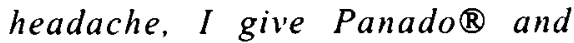
sometimes I give Lingana and Verenheid (a herb) mixed with lemon for flu and for stomach ache I give castor oil". For sore feet she uses grease oil, which is helping a lot. She smears it on her foot. For high blood, Aloe is used boiled in water and then drunk. It also helps a lot for pains. Another mother reported that she gives "anything available in the house, for example cough mixtures, Disprins ${ }^{\circledR} \quad$ (Aspirin) and even traditional herbs such as Lingana helps for flu. It works."

\section{- Take immediate action} $(21.4 \%)$

Respondents also reported that they "tried nothing" and preferred to go to the clinic immediately. One respondent reported that she would not give a family member anything before taking him to the clinic as she was scared that the registered nurses would think the health problem was her fault.

\section{- $\quad$ Religious care $(5.4 \%)$}

Some respondents preferred to include religious care in their decisions about a person needing health care. "I would also go to the Zionist Christian Church for pravers and tea. If everything tried, fail, $l$ would go to the clinic." Another respondent said that she would go to church first. They pray for her and then for her leg; they at least give her olive oil to smear on her leg.

\section{- $\quad$ Non-clinic option (5.4\%)}

Some respondents reported that they would not take a sick family member directly to the clinic, but rather to the general practitioner or the hospital. Several reasons are given, for example: "My first action is to go to the private doctor, not to the clinic because the doctor acts quickly and refer my sick one straight to the hospital. The clinic is not a good option when someone is seriously ill they take their time to help them and people working in the clinic are unapproachable."

\section{Theme 3: Assessment of the} seriousness of a health problem

Data saturation was obtained after 43

Figure 2 : Specific symptoms considered as an indication of seriousness

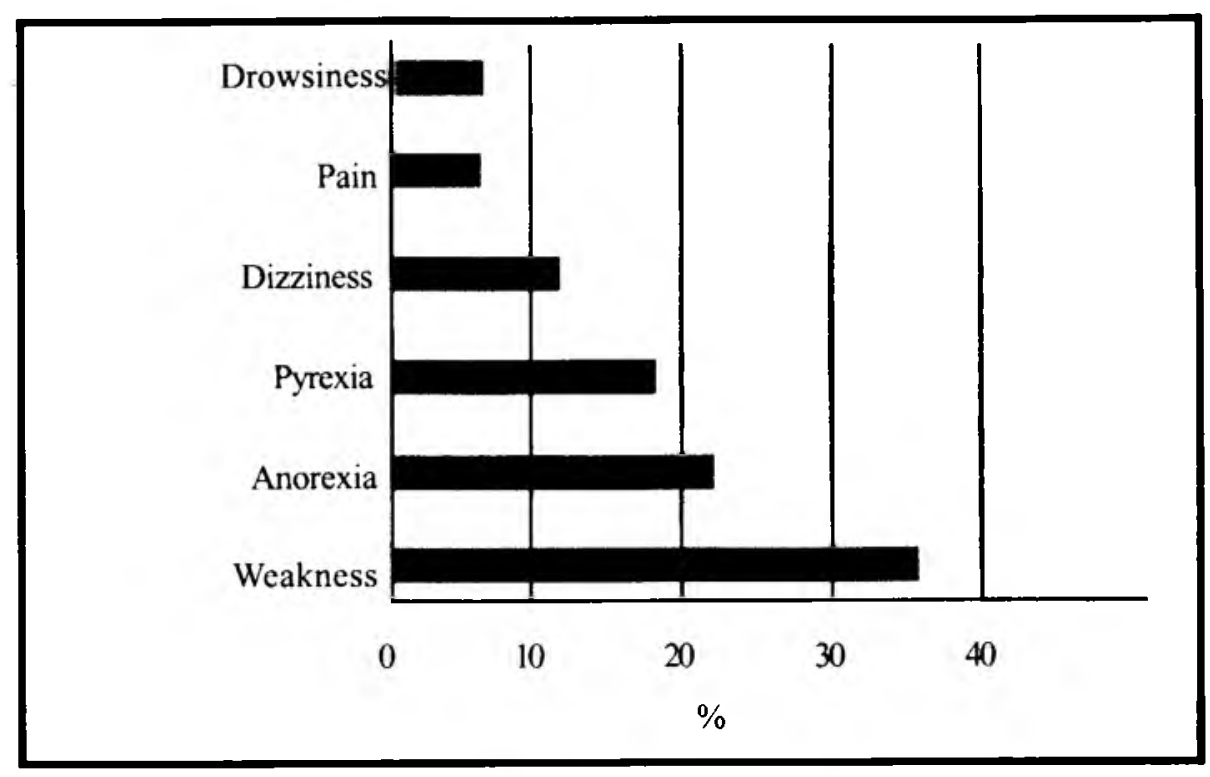

interviews.

hypertension.

Four main categories emerged from the data. The four main categories are the occurrence of specific symptoms $(68 \%)$, if self-medication is ineffective (14\%), observation first ( $11 \%$ ) and all complaints are considered serious $(7 \%)$.

The majority of the participants regarded a specific symptom or combination of symptoms as serious. The frequency of the specific symptoms is presented in Figure 2.

Weakness is most often considered as a sign of a serious health problem, often associated with the family member not wanting to eat anymore. The combination of weakness and anorexia in a child is considered as a sign of serious health problems.

"If the person cannot do anything anymore, he is taken to the clinic."

"If the person refuses food and becomes weak, then the condition is considered serious."

Only two out of ten respondents considered fever as serious. The diagnosis of fever is made with the back of the hand on the forehead of the person.

'If a child's temperature is high, I know' the person is feverish. I do that by hand touching. If the person does not have a high temperature, I know' everything is okay, the person is not seriously ill."

Dizziness $(11 \%)$ is often considered as a complication of uncontrolled
"For a person with hypertension, if I become dizzy. I know that the hypertension is now high. "

"For my high blood, when it becomes high and very uncontrollable, I will have a serious headache, become dizzy." Pain or an increase in the current level of pain as well as sleepiness is also considered to be indicative of a health problem.

The second major category is to self medicate first and if the method tried is ineffective, then the condition is considered serious. Several methods of self-medication, religious and herbal, is tried before the sick person will be taken to the clinic.

"I listen to the child's fever by hand, if the health problem does not subside after everything being tried, then the condition is considered serious."

"'If herbs did not help then I consider it serious and takes the child to the clinic."

Observation of the person before taking action is the third major category (11\%). A small group of caregivers $(7 \%)$ indicated that all complaints are considered serious

"I do not wait for any health problem to be serious, because they can see what should be done - whether the clinic can help or not."

Theme 4: Help seeking preferences for health related problems

Data saturation was obtained after 24 interviews.

Six main categories emerged from the 
Figure 3 : Help seeking preferences for health related problems

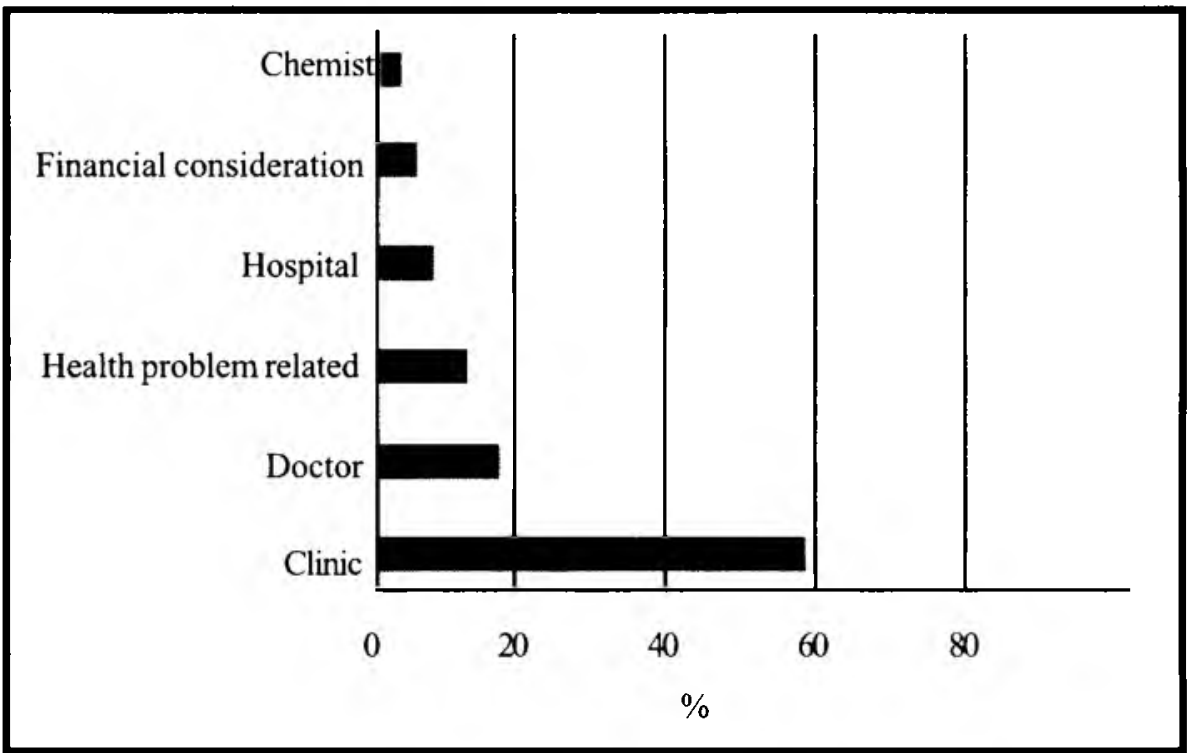

data. The respondents reported various preferences when a health problem occurs. Help seeking preferences are presented in Figure 3.

Almost six of every ten respondents preferred to use the local clinic, the reason being that the clinic is free and accessible. Some respondents $(18 \%)$ preferred to go to a general practitioner because they can afford to do so due to membership of a medical aid.

"The doctor is preferred because I have a medical aid. It is difficult to go to the clinic because I cannot stand the line." "The doctor is preferred because of $m y$ hypertension and I have a medical aid."

Some respondents $(12 \%)$ reported that their decision is based on the health problem. They would decide to go to a traditional healer, doctor, clinic or the church depending on the evaluation of the health problem. Three respondents reported that they prefer to go directly to the hospital. One respondent reported as follows:

"I prefer going to the hospital but if I do not have money, I will go to the clinic. The hospital only wants a referral letter during the week, not weekends so I can go to the hospital on Saturday."

Some respondents $(4 \%)$ make their decision in terms of the financial resources available. If they have the necessary finances, they prefer to go to a general practitioner and if not, they will go to the clinic.
Data saturation was obtained after 20 interviews.

Three main categories emerged from the data. The categories are making the decision alone $(86 \%)$, making the decision as a family $(8 \%)$ and consulting with the expanded family $(6 \%)$.

The majority of the women $(86 \%)$ reported that they decide what should happen to a family member in case of a health problem. The role of the woman of the household was taken very seriously. "As a woman, I do make the decision; a man knows nothing about children."

"I make the decision myself. I will not wait for the husband because what if the condition is serious and a child is very sick and might complicate while still decision."

A small group (2\%) reported that the decision is made as a family but not to the detriment of the person needing health care.

\section{Theme 6: Concept of time in health related problems}

Data saturation was obtained after 46 interviews.

Three main categories emerged from the data. The categories are delayed help seeking (longer than one day) (48\%), immediate help seeking (within one day) $(30 \%)$ and concept of time in case of a child $(22 \%)$.

Half of the respondents would wait more than a day, sometimes even a week or waiting for someone to make a longer to seek help.

"Not more than three days but in

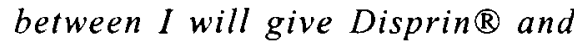
herbs."

" $A$ week then if the condition stays the same they will go to the clinic."

"2-3 days, not more than a week."

"For myself, I have high blood pressure.

I wait for the return date though I feel sick before the return date. I will drink whatever I can, I will not go to the clinic."

Three out of every ten respondents reported that they would wait less that one day. They considered any health problem as serious and no time is wasted. A person is taken to the clinic or hospital in case of a health problem. When the person in need of health care is a child, the time allowed before a decision is taken, varies. Often the child would be taken to the clinic sooner than an adult, though this was not always the case.

"Depends on the condition, but if it is a child I go straight to the clinic."

"A day is enough for a headache for" myself. If it is a child, two days is enough while I will be checking the seriousness of the condition."

"A week for myself, for a child a day is given for a trial of my' own medication then taken to the clinic."

\section{Discussion}

Several issues emerged from the research that health care providers should take cognisance of.

The role of the registered nurse in the primary health care clinic is very important. Often their behaviour, not their clinical knowledge and skills, determined the benefit the community can obtain from the proximity of the clinic (Tlebere et al., 2007:342). In addition, Mills et al. (2004:938) add that nurses in urban clinics were criticized for their attitude and treatment of the patients, rudeness, lack of confidentiality and blatant favouritism towards those that they knew or perceived to be more affluent. From the other side of the coin, Gilson and Schneider (2007:29) report that the registered professional nurses in South Africa themselves admit that they take their own frustrations out on patients and abuse the power relationship between provider and patient.

Only $58 \%$ of the women reported using the clinic as their first contact in case of a 
health problem. The rest of the group preferred any other choice available to them, very often because they were not satisfied with the services rendered at the clinic. Support for this statement is provided by Bennett, McPake \& Mills, 1997). The authors write that due to dissatisfaction with the quality of the service, where users have a choice, they frequently use private providers ranging from informal drug sellers to trained professionals. As the group of women were mostly unemployed (74\%), a valuable support system is undermined by the behaviour of the registered nurses and the lack of a steady supply of medication at the clinics. Mills et al. (2004:931) report that in low and middleincome countries, primary care are still incomplete and that the technical and user-perceived quality is often poor.

Willingness to learn is fundamental to learning and should be exploited to its fullest advantage. According to the study, seven out of ten respondents declared a willingness to learn. Health care providers should take advantage of this willingness, as adults are most interested in learning subject matter that have immediate relevance to their job or personal life. Adult learning is also problem-centered rather than contentoriented (Knowles, 1984). In addition, according to the assumptions underpinning adult learning, adult learners need to feel that they are responsible for their own decisions and to be treated as self-directed. Also, adult learners have usually had many life experiences that they consider to be the best source of learning. Adults usually want to use what they know from experiences and would like to receive acknowledgement for having the acquired knowledge. If the health education at the clinic is focussed on HIV/AIDS to the exclusion of other diseases and conditions, a valuable opportunity is lost. Of concern, is the remaining three out of ten who indicated an unwillingness to learn. There is an indication that the social environment in the clinic is not always conducive to learning.

The behavioural patterns when a person needs health care raise some concerns. Seven out of ten respondent $(68 \%)$ would self medicate first. Self-medication involves a delay in obtaining healthcare. If the self-medication consists of measures such as vomiting to reduce headache or drinking Aloe boiled in water for high blood pressure, there might be an inherent danger in these practices. The propensity to self-medicate against the background of when a health problem is considered serious, presents health promoters with a challenge. Weakness or physical inability was most often $(36 \%)$ considered as a sign of serious health problems. Only two out of ten respondents considered anorexia or pyrexia as a sign of a serious health problem. One in ten considered dizziness as a problem and then only in terms of hypertension. The health risks related to self medication are supported by Tupasi et al. (1989:82) who write that due to mothers' limited knowledge and poor diagnostic ability, the self medication may be inappropriate. Bussing et al. (2006:871) add that self care strategies are commonly used and appear to co-exist with professional therapy. They urge health care providers to explore possible self care strategies used by mothers as these may interfere with prescribed treatment.

The time interval until health care is obtained is also indicative of a possible obstacle in maintaining the health of the community. Delayed help seeking occurred in half of the group and in some respondents, the decision regarding children is dangerous. The social environment in the primary health care clinic was again mentioned as a reason for the delay.

From the results, it is clear that the woman in the household independently makes decisions regarding health related matters. This consistency provides an opportunity to develop a health intervention to teach women new practises regarding health matters.

\section{Recommendations}

As most of the women in the study sample were from one socio-cultural group namely the Tswana group, the study research should be repeated on a larger scale to ensure that a complete understanding of health care practices in the specific community. Based on this knowledge and understanding, an intervention targeting all the women in the community should be developed and implemented. Health care professionals should be made cognisant of the self care practises of mothers as the self care could either have contributed to the current health problem or may interfere with the treatment.

\section{Conclusion}

Without an understanding of the range of health care practices used by mothers in a specific community, registered professional nurses may misdiagnose a serious health problem in a patient. The misdiagnosis may result from not fully understanding the history or not asking the mother the appropriate questions. In both cases, the result could be serious for the patient.

\section{References}

BENNET, S; MCPAKE, B \& MILLS, A 1997: Private health care providers in developing countries: serving the public interest? London: Zed Books.

BRINK, HI 1996: Fundamentals of research methodology for health care professionals. Kelwyn: Juta.

BUNTON, R; NETTLETON, S \& BURROWS, R, eds. 1996: The sociology of health promotion: critical analyses of consumption, lifestyle and risk. London: Routledge.

BURNS, N \& GROVE, SK 2005: The practice of nursing research: conduct, critique \& utilization. Philadelphia: WB Saunders.

BUSSING, R; KORO-LJUNGBERG,ME; WILLIAMSON, P; GARY, FA \& GARVAN, CW 2006: What "Dr. Mom" ordered: a community-based exploratory study of parental self-care responses to children's ADHD symptoms. Social Science \& Medicine. 63(4):871-882.

CRESWELL, JW 1994: Research design: qualitative and quantitative approaches. Thousand Oakes: Sage.

DENNILI , K; KING, L\& SWANEPOEL, T 2004: Aspects of primary health care in Southern Africa. $2^{\text {nd }}$ ed. Cape Town: Oxford University Press Southern Africa

GILBERT, L \& WALKER, L 2002: Treading the path of least resistance: HIV/AIDS and social inequalities - A South African case study. (In Gilbert, T Selikow \& Walker, L Society Health and Disease: an introductory reader for health professionals. Braamfontein: Ravan).

GILSON, L \& SCHNEIDER, H 2007: Understanding health service access: concepts and experience. Global Forum 
KELLY, MP \& CHARLTON, B 1996:

The modern and post-modern in health promotion. (In Bunton, R; Nettleton, S \& Burrows, R eds. The sociology of health promotion: critical analyses of consumption, lifestyle and risk. London: Routledge).

KNOWLES, M 1984: The Adult Learner: A Neglected Species (3rd ed.). Houston, TX: Gulf Publishing.

LINCOLN, YS \& GUBA, EG 1985: Naturalistic inquiry. Newbury Park: Sage.

MILLS, A; PALMER, N; GILSON, L; MCINTRYE, D; SCHNEIDER, H; SINANOVICC, E \& WADEE, H 2004: The performance of different models of primary care provision in Southern Africa. Social Science \& Medicine, 59:941-943.

SHARP, J 1988: Introduction: Constructing social reality. (In Boonzaier, E \& Sharp, J eds. South African Keywords: the uses and abuses of political concepts. Cape Town: David Philip, pp. 1-16).

TLEBERE，P; LOVEDAY，M; MATIZIROFA, L; MBOMBO, N; DOHERTY,T; WIGTON,A; TREGER, L \& CHOPRA, M 2007: Community-based situation analysis of maternal and neonatal care in South Africa to explore factors that impact utilization of maternal health services. Journal of Midwifery and Women's Health, 52(4):340-350.

TUPASI,TE;MIGUEL,CA; TALLO,VL; BAGASAO, TM; NATIVIDAD, JN; VALENTIA, LB; DE JESUS, ME; LUPISAN, S \& MEDALLA, F 1989:

Child care practices of mothers: implications fro intervention in acute respiratory infections. Annals of Tropic Paediatrics. 9(2):82-88. 